\title{
INITIAL POSTBUCKLING BEHAVIOR OF SHEAR DEFORMABLE SYMMETRICALLY LAMINATED BEAMS
}

\author{
A. M. WAAS \\ Department of Aerospace Engineering, University of Michigan, Ann Arbor, MI 48109-2140, \\ U.S.A.
}

(Received 19 June 1991; in revised form 4 November 1991)

\begin{abstract}
Results for the initial postbuckling behavior of laminated beams are presented. Shear deformation effects are incorporated by relaxing certain assumptions of thin beam/plate theory. The analysis used is in the spirit of Koiter [Doctoral thesis, Delft, Amsterdam, English translation AFFDL-TR-70-25, (1970)], who developed asymptotic expressions for the potential energy in the neighborhood of the critical load in terms of a small parameter, which was identified as the amplitude of the buckling mode. The method adopted in the present paper is to use asymptotic expansion considerations to differential equations obtained via the principle of stationary potential energy. The effect of boundary conditions on the initial postbuckling response is studied by considering the two extreme cases of pinned-pinned and fully clamped boundary supports. An extensive parametric study is conducted to study the effects of mechanical properties, emerging as suitable non-dimensional groups from the derivation of the governing equations, on the initial postbuckling response. Finally, the effect of shape imperfections on the response of the beam is studied for certain example configurations that exhibit a limit load type of behavior.
\end{abstract}

\section{INTRODUCTION}

It is well known that classical thin plate theory, which does not account for transverse shear deformation effects, fails to yield reasonable predictions, even for appreciably large aspect ratios (span to thickness ratios), by standards established through analysis of beams/plates made of isotropic materials, when used to analyze laminated composite plates [1]. In the past decade or so, several research investigations that deal with improving classical plate theory to include effects such as shear deformation, have emerged. The results of these investigations and their importance to the field are nicely presented in recent articles by Reddy [2, 3].

Buckling and postbuckling of composite beam/plate structures is a subject of continued investigation. Amongst the most active in this area are Stein and coworkers at the NASA Langley Research Center, who have presented a series of articles aimed at developing displacement-based theories to study buckling, vibration and importantly, postbuckling behavior of laminated composite beams, plates and shell structures. Their work, and other contributions to this topic are presented in refs [4-7]. The governing field equations, boundary conditions and solution procedure are clearly explained in these articles, and, where possible comparison with experiments are reported.

A finite element study, incorporating a higher-order kinematic model with shear deformation accounted for, was recently presented by Sheinman and Adan [8]. In solving the fully non-linear governing equations, these researchers used a modification of Newton's method and a special finite difference scheme in the solution process. Results for both isotropic and anisotropic beams are presented. Reddy and coworkers [9-12] incorporated geometrical non-linearity to study stability, vibration and bending problems in laminated beams, but emphasis was not on the study of postbuckling behavior.

The present investigation is concerned with the initial postbuckling behavior of laminated beams; the analysis adopted is developed in the spirit of Koiter [13]. The assumptions of classical theory are relaxed and a linear through thickness variation in the displacement field is assumed; this necessitates the inclusion of shear deformation, through the nonvanishing through thickness shearing strain. The motivation for the assumed displacement 
field stems from the discussion on the rigorous elastic analysis presented in Stoker [14] and Novoshilov [15].

The development of the initial postbuckling analysis, as presented in Koiter [13], basically amounts to developing asymptotic expressions for the potential energy in the neighborhood of the critical load in terms of a small parameter, which is identified as the amplitude of the buckling mode. Koiter's original work [13], was extended by Budiansky and Hutchinson [16], where a formalism based on the principle of virtual work was used. The approach adopted in the present paper is to use asymptotic expansion considerations to differential equations obtained via the principle of stationary potential energy.

The expressions obtained herein, for laminated beams, are specialized, by making appropriate substitutions to the mechanical properties, to isotropic beams and the resulting expressions are found to be in agreement with previously known results. The effect of boundary conditions on the initial postbuckling response is studied by considering the two extreme cases of pinned-pinned and fully clamped boundary supports. An extensive parametric study is conducted to study the effects of mechanical properties, emerging as suitable non-dimensional groups from the derivation of the governing equations, on the initial postbuckling response. Finally, the effect of shape imperfections on the response of the beam is studicd for certain example configurations that cxhibit a limit load type of behavior.

\section{PROBLEM FORMULATION}

\section{Perfect beams}

We consider a rectangular and symmetrically laminated uniform beam of length $L$, unit breadth and thickness $h$, subjected to an axial compressive load $P$ applied at the ends. We assume that strains are small compared to unity and that planes normal to the centroidal line in the undeformed state remain plane but are not restricted to be normal to the deformed centroidal line. Further, we assume that the centroidal line of the beam remains inextensible. We choose a Lagrangian description with a fixed right-handed rectangular Cartesian frame of reference to be used, with the $X$-axis coinciding with the centroidal line of the beam in the initial undeformed state and the $Z$-axis normal to it. Let the components of the displacement of a particle positioned initially at $(X, Z)$ be denoted by $U, W$. In the Lagrangian description, the Greens strain tensor, referred to the initial configuration is used, whose components are

$$
\begin{aligned}
& E_{X}=\frac{\partial U}{\partial X}+\frac{1}{2}\left[\left(\frac{\partial U}{\partial X}\right)^{2}+\left(\frac{\partial W}{\partial X}\right)^{2}\right] \\
& E_{Z}=\frac{\partial W}{\partial Z}+\frac{1}{2}\left[\left(\frac{\partial W}{\partial Z}\right)^{2}+\left(\frac{\partial U}{\partial Z}\right)^{2}\right] \\
& E_{X Z}=\frac{1}{2}\left[\frac{\partial U}{\partial Z}+\frac{\partial W}{\partial X}+\frac{\partial U}{\partial X} \frac{\partial U}{\partial Z}+\frac{\partial W}{\partial X} \frac{\partial W}{\partial Z}\right]
\end{aligned}
$$

Consistent with the assumptions stated, we have

$$
\begin{aligned}
& U(X, Z)=U_{0}(X)-Z \frac{\mathrm{d} \Psi_{0}}{\mathrm{~d} X} \\
& W(X, Z)=W_{0}(X)+Z \frac{\mathrm{d} U_{0}}{\mathrm{~d} X} .
\end{aligned}
$$

Here, a subscript " 0 " is used to denote quantities associated with the centroidal line. The condition of inextensibility of the centroidal line renders a relation between $U_{0}(X)$ and $W_{0}(X)$ in the form

$$
2 E_{X_{0}}=\left(\frac{\mathrm{d} U_{0}}{\mathrm{~d} X}+1\right)^{2}+\left(\frac{\mathrm{d} W_{0}}{\mathrm{~d} X}\right)^{2}-1=0
$$


Substituting equation (2) in equation (1) and using equation (3), we find that the non-zero strain components are as follows:

$$
\begin{aligned}
E_{x} & =-Z\left[\frac{\Psi_{0_{x x}}+W_{0_{x}}^{2}\left(W_{0 x x}-\Psi_{0 x x}\right)}{\sqrt{1-W_{0 x}^{2}}}\right]+\frac{Z^{2}}{2}\left[\frac{\Psi_{0 x x}^{2}+W_{0 x}^{2}\left(W_{0 x x}^{2}-\Psi_{0 x x}^{2}\right)}{\left(1-W_{0 x}^{2}\right)}\right] \\
E_{z} & =\frac{1}{2}\left[\Psi_{0_{x}}^{2}-W_{0 x}^{2}\right] \\
E_{x z} & =\frac{1}{2}\left(W_{0_{x}}-\Psi_{0_{x}}\right)\left[\sqrt{\left.1-W_{0 x}^{2}\right]}+\frac{Z}{4} \frac{\mathrm{d}}{\mathrm{d} X}\left[-W_{0 x}^{2}+\Psi_{0_{x}}^{2}\right]\right.
\end{aligned}
$$

Notice that if we set $W_{0}(X)-\Psi_{0}(X) \equiv 0$, then the displacement field (2), along with equation (3), simplifies the non-linear strain components of the Greens strain tensor to the extent that the shearing strain $E_{X z}$ and the transverse normal strain $E_{Z}$ are both zero, thus reverting to simplifications of classical beam theory. Corresponding to the Greens strain tensor, we have Kirchhof's stress tensor $S_{i j}$, which will be used in the analysis. Assuming a state of plane stress in the $X Z$-plane and with the assumption on the smallness of $E_{X}$, we invoke Hooke's law taken in the simple form as suggested by Stein [6]

$$
S_{X}=\mathrm{E} E_{X}, \quad S_{z}=\mathrm{E}_{z} E_{z}, \quad S_{X Z}=2 G_{x z} E_{X z}
$$

where, the stiffnesses $E, E_{z}$ and $G_{x z}$ are thickness-averaged moduli of the laminated beam material. Next, we write the potential energy $(\Pi)$ of the beam

$$
\Pi=\frac{1}{2} \int_{0}^{L} \int_{-h / 2}^{h / 2}\left(S_{X} E_{X}+S_{Z} E_{Z}+S_{X Z} E_{X Z}\right) \mathrm{d} Z \mathrm{~d} X+P \int_{0}^{L} \frac{\mathrm{d} U_{0}}{\mathrm{~d} X} \mathrm{~d} X .
$$

$\Pi$ can be expressed in terms of $W_{0}(X)$, the lateral displacement of the centroidal line and $\Psi_{0}(X)$, and their derivatives by using equations (1), (4) and (5) in equation (6). For the sake of brevity, the subscript 0 associated with $W_{0}(X)$ and $\Psi_{0}(X)$, and their derivatives are dropped. Then, carrying out the integration in $Z$ and defining

$$
\int_{-h / 2}^{h / 2} Z^{2} \mathrm{~d} Z=I
$$

we obtain

$$
\begin{aligned}
\Pi= & \frac{E I}{2} \int_{0}^{L}\left[\Psi_{X X}^{2}\left(1-W_{X}^{2}\right)+2 \Psi_{X X} W_{X X} W_{X}^{2}\right] \mathrm{d} X+\frac{\mathrm{E}_{Z} h}{8} \int_{0}^{L}\left(\Psi_{X}^{4}-2 \Psi_{X}^{2} W_{X}^{2}+W_{X}^{4}\right) \mathrm{d} X \\
& +\frac{k \mathrm{G}_{X Z} I}{2} \int_{0}^{L}\left(W_{X}^{2} W_{X X}^{2}+\Psi_{X}^{2} \Psi_{X X}^{2}-2 W_{X} W_{X X} \Psi_{X} \Psi_{X X}\right) \mathrm{d} X \\
& +\frac{k \mathrm{G}_{X Z} h}{2} \int_{0}^{L}\left[\left(W_{X}-\Psi_{X}\right)^{2}\left(1-W_{X}^{2}\right)\right] \mathrm{d} X-\frac{P}{2} \int_{0}^{L}\left(W_{X}^{2}+\frac{1}{4} W_{X}^{4}\right) \mathrm{d} X
\end{aligned}
$$

where, we have retained all the quartic terms entering in equation (6), associated with nonquadratic terms (in $Z$ ) of the expressions for the strains, and $k$ is a shear correction factor. ( ) $x$ indicates differentiation with respect to $X$ (or $x$ in the normalized expressions to appear later) of the quantity (quantities) appearing within the parenthesis. The terms entering $\Pi$ that are the result of retaining quadratic terms (in $Z$ ) in the expression for $E_{X}$ have been neglected. Some of these terms may contribute a quartic (in $W_{0}$ ) term to $\Pi$. However, as was shown in a similar study before [17], these terms have a negligible contribution to $\Pi$. Before proceeding further, the following non-dimensional quantities are introduced:

$$
\begin{aligned}
& w=\frac{W}{L}, \quad x=\frac{X}{L}, \quad \sigma=\frac{P L^{2}}{4 \mathrm{E} I}, \quad \Pi^{*}=\frac{2 \Pi L}{\mathrm{E} I} \\
& \beta=\frac{L^{2} \mathrm{E}_{z} h}{4 \mathrm{E} I}, \quad \delta=\frac{k L^{2} \mathrm{G}_{x z} h}{\mathrm{E} I}, \quad \gamma=\frac{k \mathrm{G}_{x z}}{\mathrm{E}} .
\end{aligned}
$$


Then

$$
\begin{aligned}
\Pi^{*}= & \int_{0}^{1}\left[\psi_{x x}^{2}\left(1-w_{x}^{2}\right)+2 \psi_{x x} w_{x x} w_{x}^{2}\right] \mathrm{d} x+\beta \int_{0}^{1}\left(\psi_{x}^{4}-2 \psi_{x}^{2} w_{x}^{2}+w_{x}^{4}\right) \mathrm{d} x \\
& +\delta \int_{0}^{1}\left[\left(w_{x}-\psi_{x}\right)^{2}\left(1-w_{x}^{2}\right)\right] \mathrm{d} x+\gamma \int_{0}^{1}\left(w_{x}^{2} w_{x x}^{2}+\psi_{x}^{2} \psi_{x x}^{2}-2 w_{x} w_{x x} \psi_{x} \psi_{x x}\right) \mathrm{d} x \\
& -\frac{P}{2} \int_{0}^{1}\left(w_{x}^{2}+\frac{1}{4} w_{x}^{4}\right) \mathrm{d} x .
\end{aligned}
$$

At equilibrium, the first variation of $\Pi^{*}$ with respect to $\delta w$ and $\delta \psi$ must vanish. Thus, by setting $\delta \Pi^{*}=0$, integrating by parts and arranging terms, we obtain

$$
\delta \Pi^{*}=\int_{0}^{1} L_{w}^{N}(w) \delta w \mathrm{~d} x+\int_{0}^{1} L_{\psi}^{N}(\psi) \delta \psi \mathrm{d} x+\text { boundary terms }=0 .
$$

Since $\delta w$ and $\delta \psi$ are arbitrary in the interval $(0,1)$, we obtain the coupled ordinary differential equations governing beam equilibrium

$$
L_{w}^{N}(w)=0 \quad \text { and } \quad L_{\psi}^{N}(\psi)=0
$$

where

$$
\begin{aligned}
L_{w}^{N}(w)= & 2\left(\psi_{x x}^{2} w_{x}\right)_{x}+2\left(\psi_{x x} w_{x}^{2}\right)_{x x}-4\left(w_{x} w_{x x} \psi_{x x}\right)_{x}-\beta\left(4 w_{x}^{3}-4 w_{x} \psi_{x}^{2}\right)_{x} \\
& -\delta\left[-2 w_{x}\left(w_{x}-\psi_{x}\right)^{2}+2\left(1-w_{x}^{2}\right)\left(w_{x}-\psi_{x}\right)\right]_{x}+\gamma\left(2 w_{x}^{2} w_{x x}-2 \psi_{x} \psi_{x x} w_{x}\right)_{x x} \\
& +2 \gamma\left(\psi_{x} \psi_{x x} w_{x x}-w_{x} w_{x x}^{2}\right)_{x}+4 \sigma\left(2 w_{x}+w_{x}^{3}\right)_{x}
\end{aligned}
$$

and

$$
\begin{aligned}
L_{\psi}^{N}(\psi)= & 2\left[\psi_{x x}\left(1-w_{x}^{2}\right)\right]_{x x}+2\left(w_{x}^{2} w_{x x}\right)_{x x}-4 \beta\left(\psi_{x}^{3}-w_{x}^{2} \psi_{x}\right)_{x}+2 \delta\left[\left(w_{x}-\psi_{x}\right)\left(1-w_{x}^{2}\right)\right]_{x} \\
& +2 \gamma\left(\psi_{x}^{2} \psi_{x x}-w_{x} \psi_{x} w_{x x}\right)_{x x}-2 \gamma\left(\psi_{x} \psi_{x x}^{2}-w_{x} w_{x x} \psi_{x x}\right)_{x} .
\end{aligned}
$$

Clearly, $w(x)=\psi(x)=0$ is a solution of equation (12) for all values of $\sigma$. The value of $P$ corresponding to the smallest value of $\sigma$ (designated $\sigma_{0}$ ) for which non-trivial solutions of arbitrarily small amplitude exist is identified as the buckling load. Let us perturb equation (12) about the state $\left(\sigma=\sigma_{0}, w(x)=0, \psi(x)=0\right)$. Thus, let

$$
\begin{aligned}
\sigma & =\sum_{n=0}^{\infty} \varepsilon^{n} \sigma_{n} \\
w(x) & =\sum_{n=1}^{\infty} \varepsilon^{n} w_{n}(x) \\
\psi(x) & =\sum_{n=1}^{\infty} \varepsilon^{n} \psi_{n}(x) .
\end{aligned}
$$

Here, $\left(\varepsilon w_{1}, \varepsilon \psi_{1}\right)$ is the buckling mode with shape $\left[w_{1}(x), \psi_{1}(x)\right]$ and small amplitude $\varepsilon$. Substituting equation (13) into equation (12) and grouping together terms according to ascending powers of $\varepsilon$, we obtain

$$
\begin{array}{ll}
\varepsilon^{1} & L_{1}\left(w_{1}\right)+L_{2}\left(\psi_{1}\right)=0 \\
\varepsilon^{2} & L_{3}\left(w_{1}\right)+L_{1}\left(\psi_{1}\right)=0 \\
L_{1}\left(w_{2}\right)+L_{2}\left(\psi_{2}\right)=0 \\
L_{3}\left(w_{2}\right)+L_{1}\left(\psi_{2}\right)=-4 \sigma_{1} w_{1 \times x} \\
\varepsilon^{3} \quad L_{1}\left(w_{3}\right)+L_{2}\left(\psi_{3}\right)=\text { RHS } 1 \\
& L_{3}\left(w_{3}\right)+L_{1}\left(\psi_{3}\right)=\text { RHS } 2
\end{array}
$$


where

$$
\begin{aligned}
L_{1}()= & \delta()_{x x} \\
L_{2}()= & ()_{x x x x}-\delta()_{x x} \\
L_{3}()= & -\delta()_{x x}+4 \sigma_{0}()_{x x} \\
\text { RHS1 }= & \left(\psi_{1_{x x}} w_{1_{x}}^{2}\right)_{x x}-\left(w_{1_{x x}} w_{1_{x}}^{2}\right)_{x x}+2 \beta\left(\psi_{1_{x}}^{3}-\psi_{1_{x}} w_{1_{x}}^{2}\right)_{x}-\delta\left[-w_{1_{x}}^{2}\left(w_{1_{x}}-\psi_{1_{x}}\right)\right]_{x} \\
& \quad-\gamma\left(\psi_{1_{x}}^{2} \psi_{1_{x x}}-w_{1_{x}} \psi_{1_{x}} w_{1_{x x}}\right)_{x x}+\gamma\left(\psi_{1_{x}} \psi_{1_{x x}}^{2}-w_{1_{x}} w_{1_{x x}} \psi_{1_{x x}}\right)_{x}
\end{aligned}
$$

and

$$
\begin{aligned}
\text { RHS2 }= & -\left(\psi_{1_{x x}}^{2} w_{1_{x}}\right)_{x}-w_{1_{x}}^{2} \psi_{1_{x x x x}}-2 w_{1_{x}} w_{1_{x x}} \psi_{1_{x x x}}+2 \beta\left[w_{1_{x}}\left(w_{1_{x}}^{2}-\psi_{1_{x}}^{2}\right)\right]_{x} \\
& +\delta\left[\left(w_{1_{x}}-\psi_{1_{x}}\right)\left(w_{1_{x}} \psi_{1_{x}}\right)-2 w_{1_{x}}^{2}\right]_{x}-\gamma\left[w_{1_{x}}\left(w_{1_{x}} w_{1_{x x}}-\psi_{1_{x}} \psi_{1_{x x}}\right)\right]_{x x} \\
& -\gamma\left[\psi_{1_{x}} \psi_{1_{x x}} w_{1_{x x}}-w_{1_{x}} w_{1_{x x}}^{2}\right]_{x}-4 \sigma_{1} w_{2_{x x}}-4 \sigma_{2} w_{1_{x x}}-2 \sigma_{0}\left[w_{1_{x}}^{3}\right]_{x} .
\end{aligned}
$$

The coupled sets of equations for each order of $\varepsilon$ can be simplified by eliminating one of the variables $w(x)$ or $\psi(x)$. Then we obtain a reduced set of equations for a single variable. Eliminating $\psi(x)$, we arrive at the following set of equations:

where

$$
\begin{array}{ll}
\varepsilon^{1} & L_{r}\left(w_{1}\right)=0 \\
\varepsilon^{2} & L_{r}\left(w_{2}\right)=-4 \delta \sigma_{1} w_{1_{x x}}+4 \sigma_{1} w_{1_{x x x x}} \\
\varepsilon^{3} & L_{r}\left(w_{3}\right)=\delta\left[\text { RHS1 }+ \text { RHS2] }-[\text { RHS } 2]_{x x}\right.
\end{array}
$$

$$
\text { ... etc. }
$$

$$
L_{r}()=\left(\delta-4 \sigma_{0}\right)()_{x x x x}+4\left(\sigma_{0} \delta-1\right)()_{x x}+4 \delta() \text {. }
$$

The first of these equations correspond to classical buckling of a shear deformable symmetrically laminated beam. The solution of this equation for appropriate boundary conditions at the two ends $x=0$ and $x=1$, will result in an expression that characterizes the effect of shear deformation on the buckling load. Several examples are presented later. Solution of successive equations corresponding to higher orders of $\varepsilon$ will yield the response of the beam in the initial stages of postbuckling. Such information is crucial, since the slope of the initial postbuckling response path of the perfect beam yields information on the degree of imperfection sensitivity of the beam structure [18]. In addition, the present solution can be used to check the validity of a fully non-linear numerical solution of a beam structure which is commonly carried out by using the finite element method [8], or by other techniques such as finite differences. The above formulation is next applied to several examples to demonstrate its purpose.

\section{ANALYSIS}

\section{Simply supported beams}

To demonstrate our results, we first consider simply supported beams. For this case, two example problems are presented. First, an isotropic beam is analyzed for which $E=E_{z}$ and $\mathrm{G}_{x z}=\frac{\mathrm{E}}{2(1+v)}$, where $v$ is Poisson's ratio of the beam material and $\mathrm{E}$ the Young's modulus.

Turning our attention to the reduced set of equations, and considering the lowest-order equation $L_{r}\left(w_{1}\right)=0$, and then using the first set of equations of the sequence (14), we find that

$$
\begin{aligned}
& w_{1}(x)=A \sin (p x)+B \cos (p x)+C x+D \\
& \psi_{1}(x)=\hat{A} \sin (p x)+\hat{B} \cos (p x)+\hat{C} x+\hat{D}
\end{aligned}
$$

where, the relation between $A$ and $\hat{A}, B$ and $\hat{B}$ etc. are obtained through the coupled equations given in the first of the sequence (14), and $p$ is defined as

$$
p^{2}=\frac{4 \sigma_{0} \delta}{\delta-4 \sigma_{0}} \text {. }
$$


The unknowns $A, B, C$ and $D$ are determined from the boundary conditions, obtained from the boundary terms arising in equation (11), $w_{1}(0)=\psi_{1 x x}(0)=w_{1}(1)=\psi_{1_{x x}}(1)=0$. For purposes of normalizing, we set $w_{1}\left(\frac{1}{2}\right)=1$. Then we find $w_{1}(x)=\sin (p x), \psi_{1}(x)=s \sin (p x)$, where, $s=\frac{1}{1-\left(\frac{4 \sigma_{0}}{\delta}\right)}$, and $\sin (p)=0$ as the condition to determine the buckling load. Thus, $p=n \pi$. From this equation and with the definition of $p$, we obtain

$$
\sigma_{0}=\frac{n^{2} \pi^{2}}{4\left(1+\frac{n^{2} \pi^{2}}{\delta}\right)}
$$

In equation (20), if we take the limit $\delta \rightarrow \infty$, we recover $\sigma_{0}=\frac{n^{2} \pi^{2}}{4}$, yielding $P=\frac{n^{2} \pi^{2} E_{l}}{L^{2}}$, which corresponds to classical buckling loads predicted from Bernoulli-Navier beam theory. Now, $\delta=12 k\left(\frac{L}{h}\right)^{2}\left(\frac{\mathrm{G}_{x z}}{\mathrm{E}}\right)$, and for a beam of rectangular cross-section, $k=\frac{5}{6}$ as shown in Reissner [19]. Noting the relation between $G_{x z}$ and $E$ for isotropic materials $\delta$ reduces to $\delta=\frac{5}{(1+v)}\left(\frac{L}{h}\right)^{2}$. With these simplifications affected, relation (20) shows that the buckling load is a function of the beam materials Poisson's ratio and the square of the beam aspect ratio $\left(\frac{L}{h}\right)$.

The rest of the sequence of equations (14), can be solved by appealing to the boundedness of $w(x), \psi(x)$ and suppressing secular terms. In doing so, we obtain successively $\left(\sigma_{1}, w_{2}, \psi_{2}\right)$, $\left(\sigma_{2}, w_{3}, \psi_{3}\right) \ldots$ etc. Thus, we obtain

$$
\begin{aligned}
w(x) & =\varepsilon \sin (p x)+\varepsilon^{3} w_{3}(x) \\
\psi(x) & =\varepsilon \sin (p x)+\varepsilon^{3} \psi_{3}(x) \\
\sigma & =\frac{\pi^{2} \delta}{4\left(\delta+\pi^{2}\right)}+\varepsilon^{2} \sigma_{2}
\end{aligned}
$$

where $w_{2}=\psi_{2}=\sigma_{1}=0, s=\frac{1}{1-\left(\frac{4 \sigma_{0}}{\delta}\right)}$ and

$$
\begin{aligned}
& \sigma_{2}=\frac{1}{4\left(1+\frac{p^{2}}{\delta}\right)} \frac{1}{\delta}\left(s R_{1}+R_{2}+\frac{1}{s} R_{3}+\frac{1}{s^{2}} R_{4}\right) \\
& R_{1}=\frac{\delta \gamma p^{4}}{2}+\frac{3}{2} \beta \delta p^{2} \\
& R_{2}=-p^{6}\left(\frac{1}{4}+\frac{\gamma}{2}\right)-p^{4}\left(\delta+\frac{3 \beta}{2}+\frac{\gamma \delta}{2}\right)-p^{2}\left(\frac{3 \beta \delta}{2}+\frac{3 \delta^{2}}{4}\right) \\
& R_{3}=p^{6}\left(\frac{3}{4}\right)-p^{4}\left(\frac{-9 \delta}{4}+\frac{\gamma \delta}{2}-\frac{\delta}{2}\right)-p^{2}\left(\frac{3 \beta \delta}{2}-\frac{3 \delta^{2}}{2}\right) \\
& R_{4}=p^{6}\left(\frac{\gamma}{2}\right)-p^{4}\left(\frac{5 \delta}{4}-\frac{\gamma \delta}{2}-\frac{3 \beta}{2}+\frac{3 \sigma_{0}}{2}\right)-p^{2}\left(\frac{3 \delta^{2}}{4}-\frac{3 \beta \delta}{2}+\frac{3 \sigma_{0} \delta}{2}\right) .
\end{aligned}
$$

Expressions (22), characterize the initial stages of postbuckling of the beam. The coeffcient $\sigma_{2}$ in equation (21), contains the parameters that influence the initial slope of the postbuckling path due to the incorporation of shear deformation effects. As a check we consider the limit $\delta \rightarrow \infty$, in the expression for $\sigma$ in equation (21). In this limit we obtain

$$
\frac{\sigma}{\sigma_{0}}=1+\varepsilon^{2}\left(\frac{\pi^{2}}{8}\right)
$$


Expression (23) has been derived in Dym [18], who considered the initial postbuckling behavior of pinned Bernoulli-Navier beams. Thus, expressions which characterize the initial postbuckling response of the beam as derived in the present investigation incorporating shear deformation effects, reduces to the results presented in Dym [18], in the limit $\delta \rightarrow \infty$; this limit resembles beams of infinite shear rigidity as postulated in classical Bernoulli-Navier beam theory.

\section{Clamped beams}

The effect of boundary conditions on the initial postbuckling response of a beam structure can be conveniently addressed by studying the two extreme cases of pinned ends and fully clamped ends. For the fully clamped case, equation (18) is subjected to $w_{1}(0)=w_{1}(1)=\psi_{1_{x}}(0)=\psi_{1_{x}}(1)=0$. Then the buckling loads are given by the two equations $\sin \left(\frac{p}{2}\right)=0$ and $\tan \left(\frac{p}{2}\right)=\left(\frac{p}{2}\right)$. From these, the smallest non-trivial solution is obtained as $p=2 \pi$, and $w_{1}(x)=\cos (p x)-1, \psi_{1}(x)=s \cos (p x)-1$, where $w_{1}\left(\frac{1}{2}\right)=1$ has been used for purposes of normalization. Using $p=2 \pi$, in conjunction with equation (19), we obtain an expression for the buckling load as

$$
\sigma_{0}=\frac{\pi^{2}}{\left(1+\frac{4 \pi^{2}}{\delta}\right)}
$$

In equation (24), if we consider the limit $\delta \rightarrow \infty$, we obtain the result for the classical buckling load predicted using Bernoulli-Navier beam theory; $\sigma_{0}=\pi^{2}$. To obtain $\sigma_{2}$ for this case, the sequence of equations (14), are solved in a similar manner as for the pinned-pinned case and doing so, the following results are obtained:

$$
\begin{aligned}
w(x) & =\varepsilon[\cos (p x)-1]+\varepsilon^{3} w_{3}(x) \\
\psi(x) & =\varepsilon s[\cos (p x)-1]+\varepsilon^{3} \psi_{3}(x) \\
\sigma & =\frac{\pi^{2} \delta}{\left(\delta+4 \pi^{2}\right)}+\varepsilon^{2} \sigma_{2}
\end{aligned}
$$

where $w_{2}=\psi_{2}=\sigma_{1}=0, s=\frac{1}{1-\left(\frac{4 \sigma_{0}}{\delta}\right)}$ and

$$
\begin{aligned}
& \sigma_{2}=-\frac{1}{4\left(\delta+p^{2}\right)}\left[p^{6} Q_{1}+p^{4}\left(\delta Q_{2}+Q_{3}\right)+p^{2} Q_{4}\right] \\
& Q_{1}=-\frac{3}{4 s}+\frac{1}{4 s^{2}}-\frac{\gamma}{2} \\
& Q_{2}=-\frac{\gamma}{2}\left(1-\frac{1}{s}-\frac{1}{s^{2}}+\frac{1}{s^{3}}\right)-\frac{1}{4}-\frac{1}{2 s}+\frac{1}{4 s^{2}} \\
& Q_{3}=\frac{3 \beta}{2}\left(\frac{1}{s^{2}}-1\right)+\frac{3 \delta}{4}\left(\frac{1}{s}-1\right)\left(\frac{1}{s}-2\right)+\frac{3 \sigma_{0}}{2} \\
& Q_{4}=\frac{3 \beta \delta}{2 s}+\frac{3 \delta^{2}}{4 s}+\frac{3 \beta \delta}{2 s^{2}}+\frac{3 \delta^{2}}{4}\left(\frac{1}{s}-1\right)\left(\frac{1}{s}-2\right)-3 \beta \delta-\frac{3 \delta^{2}}{4}+\frac{3 \sigma_{0} \delta}{2} .
\end{aligned}
$$

As before, considering the limit $\delta \rightarrow \infty$, we obtain, for fully clamped beams,

$$
\frac{\sigma}{\sigma_{0}}=1+\varepsilon^{2}\left(\frac{\pi^{2}}{2}\right)
$$

To the best knowledge of the author, expression (27) has not been derived before. It is interesting to note that the coefficient of the quadratic terms in equations (23) and (27), scale by the same ratios as the fundamental buckling loads, the latter case for clamped boundary conditions having a higher value. 


\section{KESULTS AND DISCUSSION}

Figure 1 (corresponding to equation (20) with $n=1$, and equation (24) with $n=1$ ) is plotted to demonstrate the effect of shear deformation on the column buckling loads for the pinned-pinned and fully clamped cases. From this figure, we see that corrections to the buckling load due to shear deformation effects are significant only for beam aspect ratios $\frac{L}{h}<10$, a result which has been established before [20]. As can be seen from this figure, this estimate, however, is strictly correct only for the pinned-pinned case; since for the clamped case a realistic regime for not considering shear deformation effects would be $\frac{L}{h}>20$. In addition, since the mechanical properties entering in the definition of $\delta$ are fixed for isotropic beams, the aspect ratio is the only parameter influencing the effect of shear on the buckling load.

In Fig. 2(a), we present the postbuckling paths of pinned beams for different values of aspect ratio $\left(\frac{L}{h}\right)$. Corresponding results for the clamped case are shown in Fig. 2(b). As expected both situations capture the effect of decreasing stiffness, immediately proceeding buckling, thus, mimicking the predictions of a full elastic calculation. In addition, the clamped case produces a 'stiffer" postbuckling path, as one would expect intuitively. To exemplify our results for the case of laminated beams, we choose to study the example problem presented in Stein [6]. This structure has a lay-up [0/90/0 $]_{\mathrm{T}}$ and possesses the following material properties; $E=19.0 \times 10^{6} \mathrm{psi}, \mathrm{E}_{z}=1.89 \times 10^{6} \mathrm{psi}$. The beam thickness $(h)$ is 0.283 in. and $\frac{L}{h}=10$. There is some uncertainty in the value of the shear modulus $G_{x z}$; the procedure to determine this value for laminated beams is not well established and in many cases the results from a simple experiment are used to ascertain $G_{x z}$. Following the suggestion in ref. [6], we use a range of values for $G_{x z}$ that vary between $0.2 \times 10^{6}$ psi to $0.9 \times 10^{6} \mathrm{psi}$. Using these values, the non-dimensional constants $\gamma, \beta$ and $\delta$ for this example are

$$
\begin{aligned}
& \delta=0.105\left(\frac{L}{h}\right)^{2} \leftrightarrow 0.474\left(\frac{L}{h}\right)^{2} \\
& \beta=0.298\left(\frac{L}{h}\right)^{2} \\
& \gamma=0.009 \leftrightarrow 0.039 .
\end{aligned}
$$

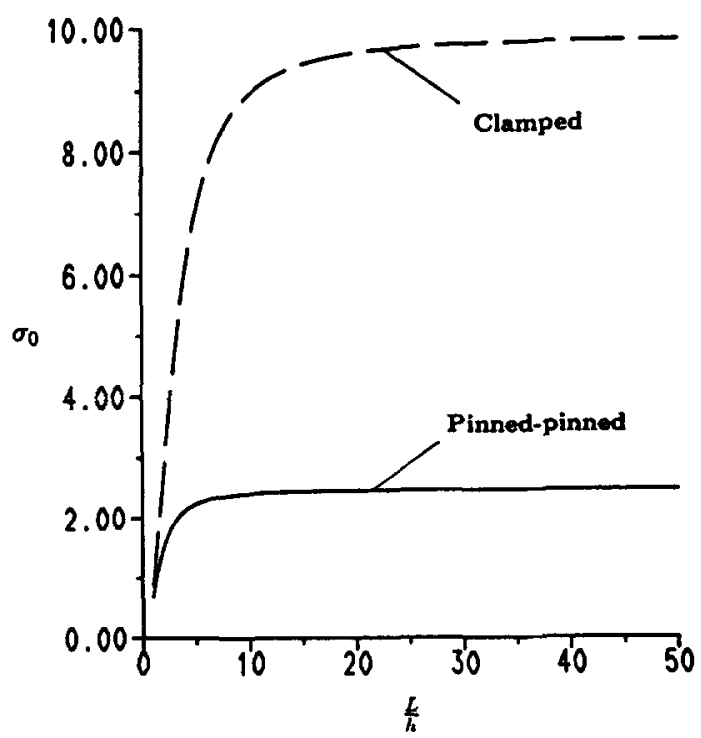

Fig. 1. The effect of shear deformation for pinned-pinned and clamped isotropic beams. 


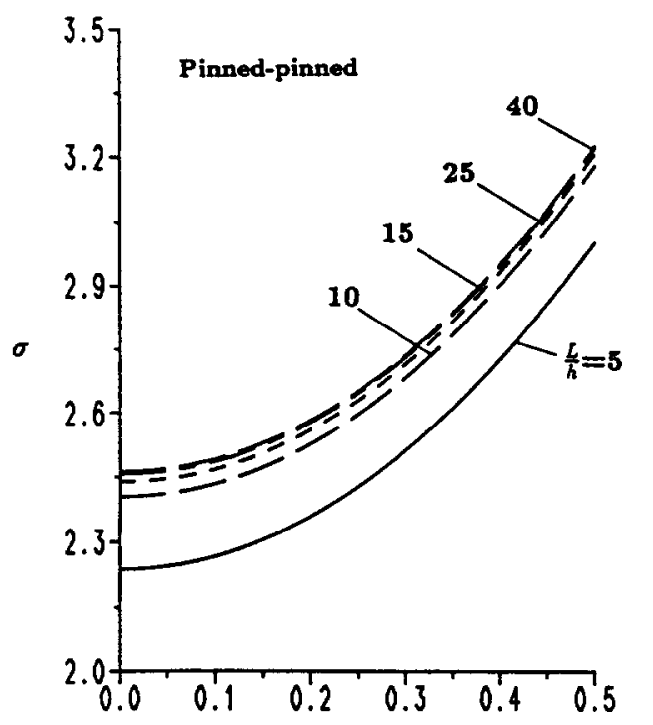

(a)

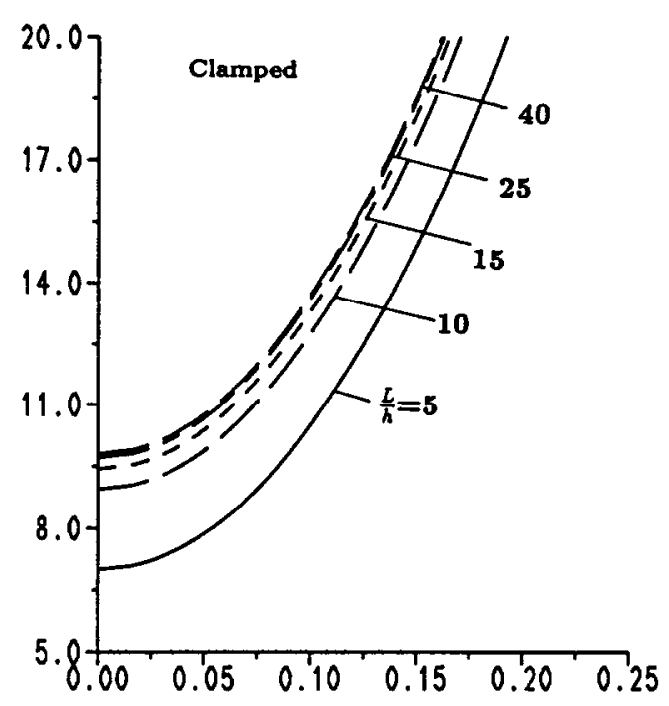

(b)

Fig. 2. (a) Postbuckling paths for pinned-pinned isotropic beams. (b) Postbuckling paths for clamped isotropic beams.
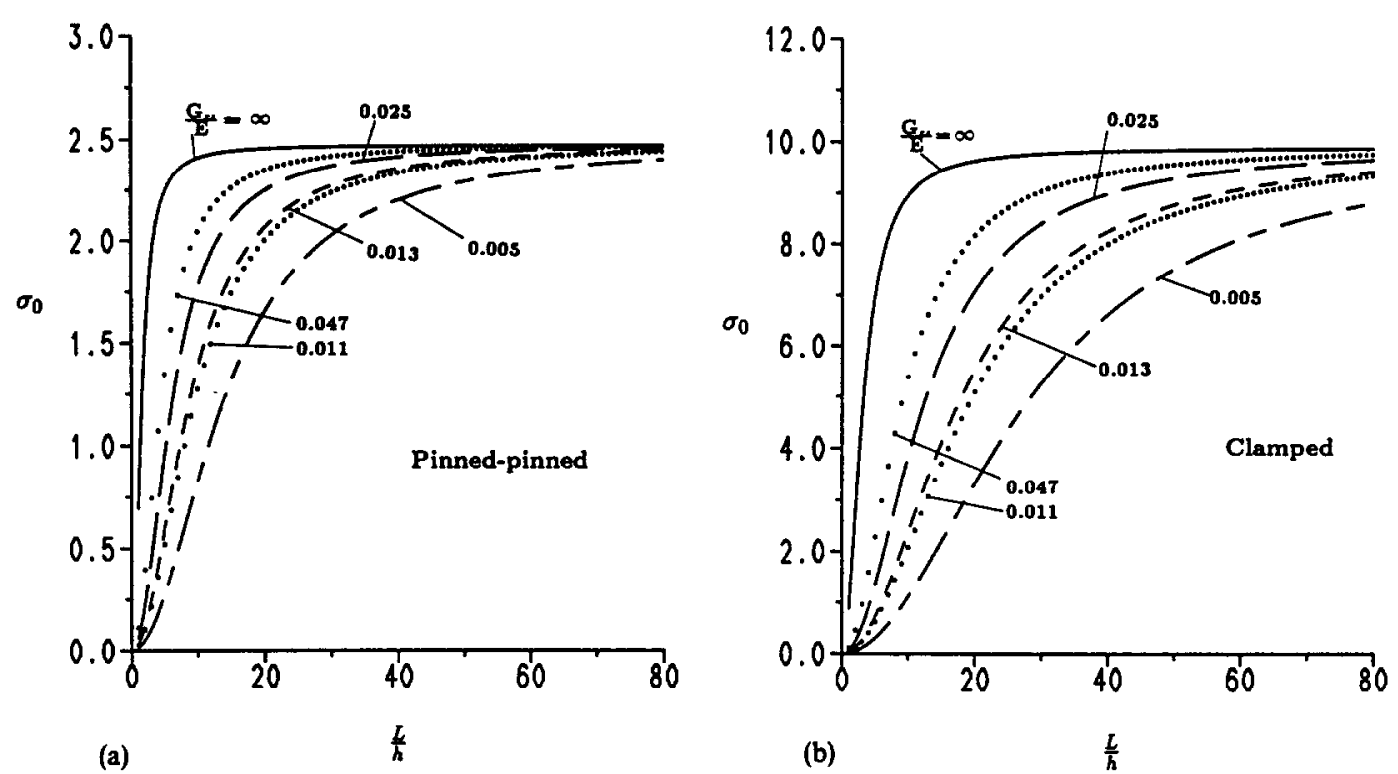

Fig. 3. (a) The effect of shear deformation for pinned-pinned laminated beams. (b) The effect of shear deformation for clamped laminated beams.

The variation of the buckling load as a function of beam aspect ratio is shown in Fig. 3(a) and (b). (In what follows, a figure number that ends with "a" corresponds to pinned-pinned conditions and one that ends with " $b$ " is for the corresponding fully clamped case.) Unlike for the isotropic case before, the buckling load additionally depends on the properties ratio $\frac{G_{x z}}{E}$. Thus, in these figures we have presented results for values of this parameter ranging from zero (a value of infinity corresponds to Bernoulli-Navier beam theory for isotropic and laminated beams) to two hundred, for values $\infty, \frac{1}{40}, \frac{1}{80}$ and $\frac{1}{200}$. Included in these 
figures are the two curves corresponding to the example problems being studied; a three layer laminated beam $[0 / 90 / 0]_{\mathrm{T}}$ (referred to as TLB), with material properties and geometry given before. The curves corresponding to this beam are shown with dots. Because of the uncertainty in $G_{x z}$, the two extreme values of $\frac{G_{x z}}{E}=0.01$ and $\frac{G_{x z}}{E}=0.1$ are used to compute the data for plotting the curves. The coupled influence of geometry (via the ratio $\left.\frac{L}{h}\right)$ and material properties via the ratio $\frac{G_{x z}}{E}$, is clear from this figure. For example, for the

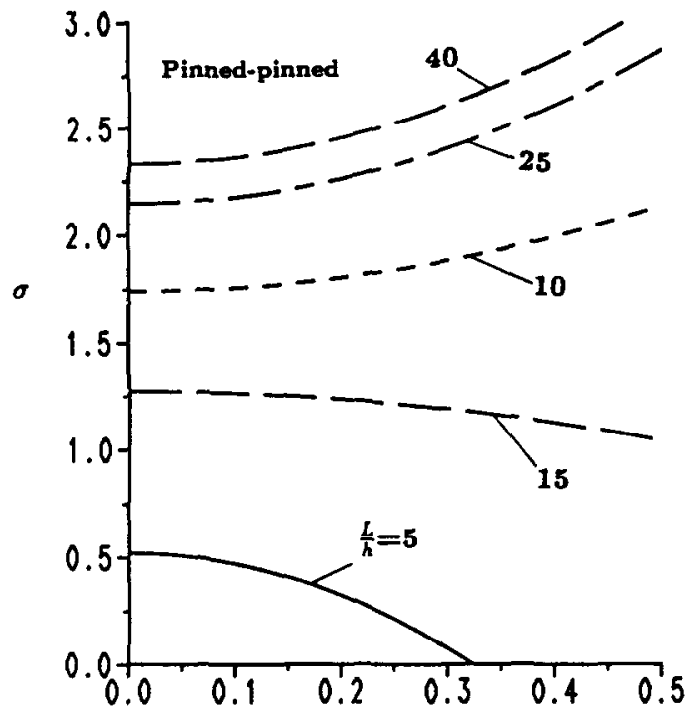

(ai)

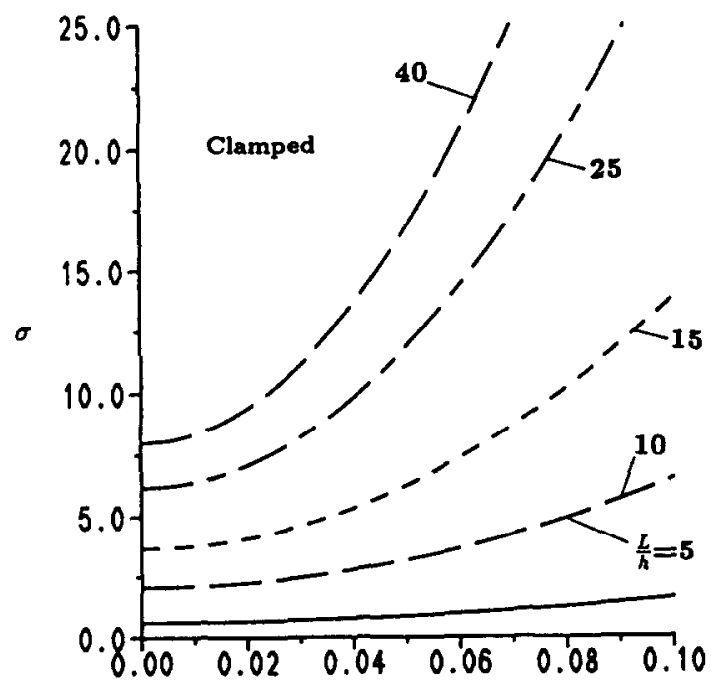

(bi)

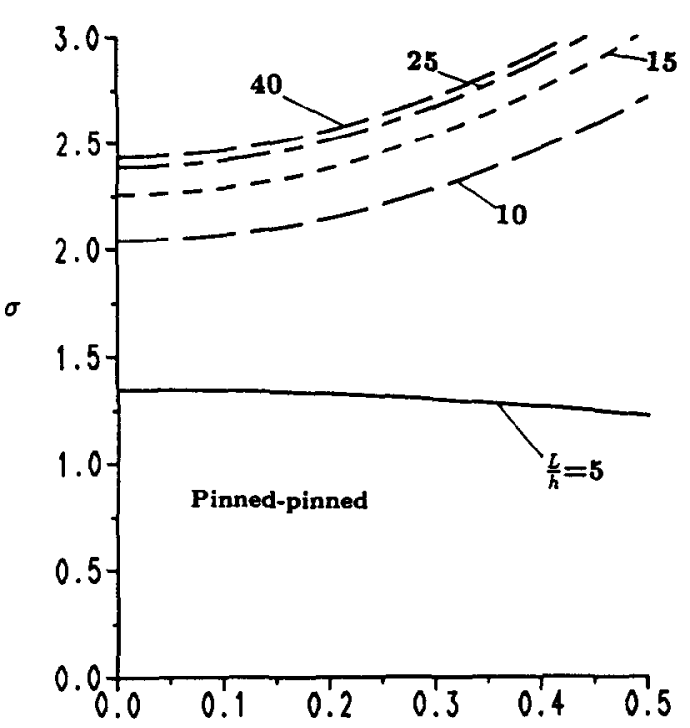

(aii)



(bii)

Fig. 4. (ai) Postbuckling paths for pinned-pinned TLB example, with $\frac{G_{x z}}{E}=0.011, k=\frac{5}{6}$.

(aii) Postbuckling paths for pinned-pinned TLB example, with $\frac{G_{x z}}{E}=0.047, k=\frac{5}{6}$.

(bi) Postbuckling paths for clamped TLB example, with $\frac{G_{x z}}{E}=0.011, k=\frac{3}{6}$. (bii) Postbuckling paths for clamped TLB example, with $\frac{\mathrm{G}_{x z}}{\mathrm{E}}=0.047, k=\frac{5}{6}$. 
TLB, with an aspect ratio of 20 , and for pinned conditions, the normalized buckling load $\sigma_{0}$ is approximately between 2.0 and 2.35 , whereas classical theory predicts 2.45 . Analogous values for the clamped case are $5.25,8.25$ and 9.6 , respectively. Thus, significant differences between the two predictions, with and without shear deformation effects are encountered; the discrepancies being larger for the clamped case. From these figures a general trend is evident. The smaller the ratio $\frac{\mathrm{G}_{x z}}{\mathrm{E}}$, the higher the value of $\frac{L}{h}$, before which, shear deformation effects have to be included for a reasonable prediction. We consider next the postbuckling path for the TLB with varying aspect ratio $\left(\frac{L}{h}\right)=5-40$. These are shown in Fig. 4(ai), (aii), (bi) and (bii). A rather surprising result corresponding to the pinned-pinned case and for the "stubby" beam of $\frac{L}{h}=5$, is evident from Fig. 4(ai) and (aii). Unlike the corresponding isotropic counterpart, the slope of the postbuckling path in the immediate vicinity of the buckling load is negative. This implies that the corresponding geometrically imperfect structure will exhibit a limit load-type behavior, which is uncharacteristic of beam response. The result of a negative slope holds true for the two extreme cases of properties ratios considered (see equation (28) for the mechanical properties, which were obtained from ref. [6]), as given in Stein [6]. However, the corresponding case with clamped conditions does not show such a trend. As the beam aspect ratio is increased, the postbuckling response shows a lower but positive stiffness. Comparison of the results presented in these figures with those of Fig. 2(a) shows that incorporation of shear deformation effects have resulted in a drastic change in the postbuckling response of a low aspect ratio laminated beam, demonstrated via the TLB example. In addition, this example demonstrates the effect of boundary conditions on the postbuckling response. The same beam structure, when supported with hinged edge conditions behaves quite differently when fully clamped.

From the expressions for the postbuckling response, equations (21) and (25), it is evident that the magnitude, and sign, of the coefficient of the quadratic term, $\sigma_{2}$, dictates the response of the structure in the immediate vicinity of the buckling load. Thus, a parametric study of $\sigma_{2}$ was carried out. In Fig. 5(ai) and (bi), the effect of $\frac{\mathrm{G}_{x z}}{\mathrm{E}}$ on $\sigma_{2}$ is presented for a fixed ratio $\frac{E_{z}}{E}=0.1$. This latter value for $E_{z}$ is a good estimate for and covers a wide range of materials that are used to construct laminates, for example, in the aerospace industry. Each curve in these figures corresponds to a particular value of beam aspect ratio. From Fig. 5(ai), it is seen that, for some range of $\frac{G_{x z}}{E}$, beams of aspect ratios, 5-10 exhibit a negative $\sigma_{2}$. The trend is very similar qualitatively and quantitatively, in Fig. 5(aii), which is a similar plot to that of Fig. 5(ai), for $\frac{E_{z}}{E}=1.0$. Thus, for pinned-pinned beams $\sigma_{2}$ is insensitive to changes in the through-thickness modulus $\mathrm{E}_{\mathbf{z}}$. Corresponding plots for the clamped case are shown in Fig. 5(bi) and (bii). In these, $\sigma_{2}$ is always positive for the range of aspect ratios studied; the particular value increasing with increasing aspect ratio. Unlike the pinned-pinned case, however, $\sigma_{2}$ is found to be sensitive to the value of $E_{2}$. It is also to be noted that for the pinned-pinned case, $\sigma_{2}$, was an increasing function of $\frac{G_{x z}}{E}$; this trend is reversed for the clamped case. Since it was established that $\sigma_{2}$ is insensitive to the value of $E_{z}$ for the pinned-pinned case, its influence on $\sigma_{2}$ was not investigated further. Figure $6(\mathrm{bi})$ and (bii), show the effect of $\frac{E_{z}}{E}$ on $\sigma_{2}$ for clamped beams. For very small values of $\frac{E_{x}}{E}$ and for small aspect ratios, it is found that $\sigma_{2}$ does attain negative values for both cases of $\frac{G_{x z}}{E}=0.01$ and 0.1. Thus, unlike pinned-pinned beams, clamped beams do show a change in the initial postbuckling behavior as a function of through-thickness modulus $\frac{E_{z}}{E}$. Finally, before 


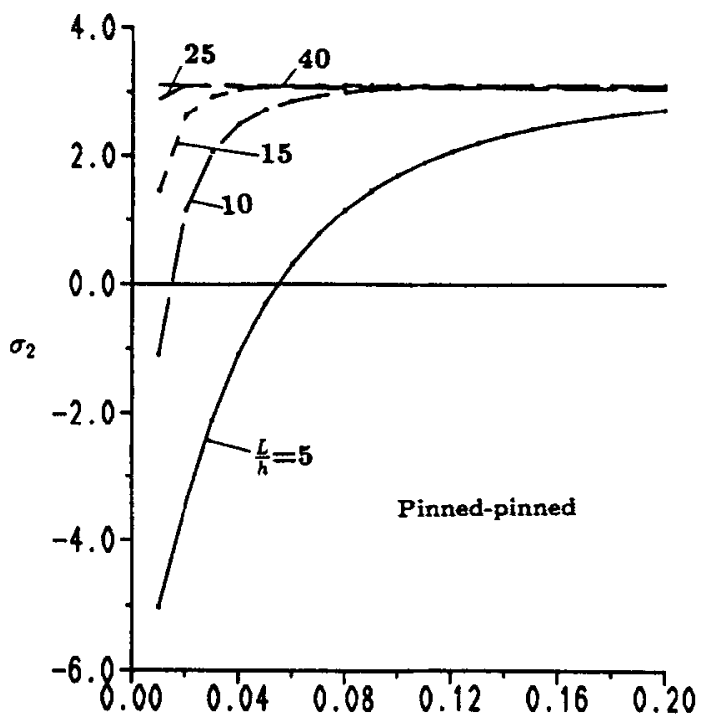

(ai)

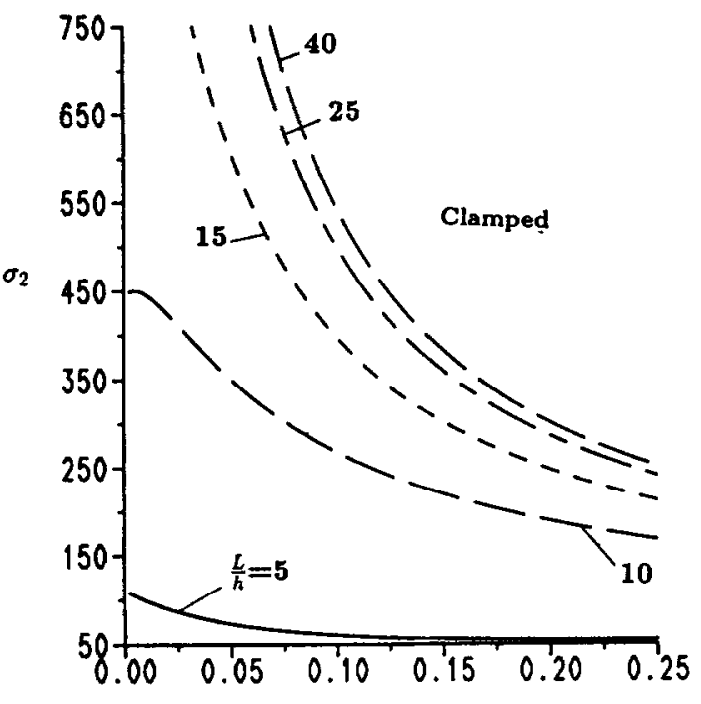

(bi)

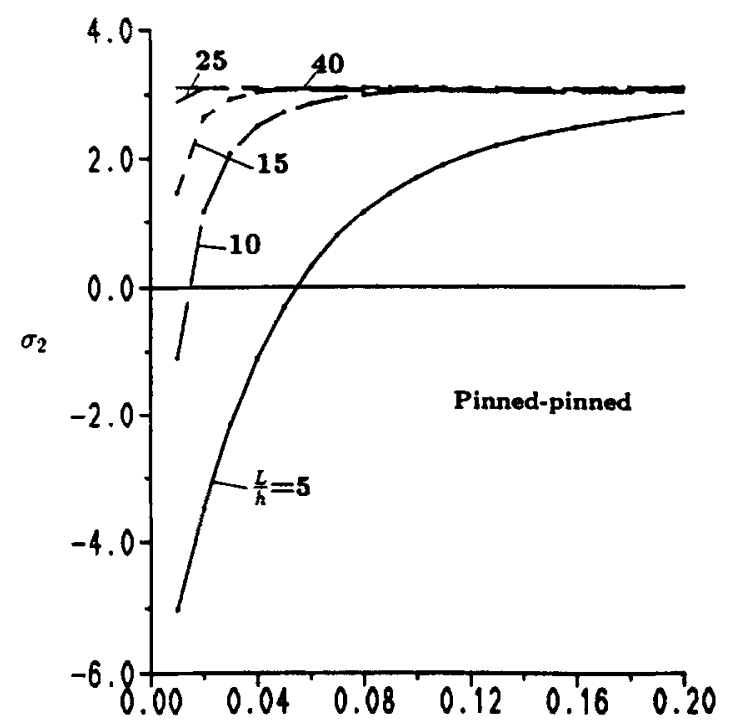

(aii)

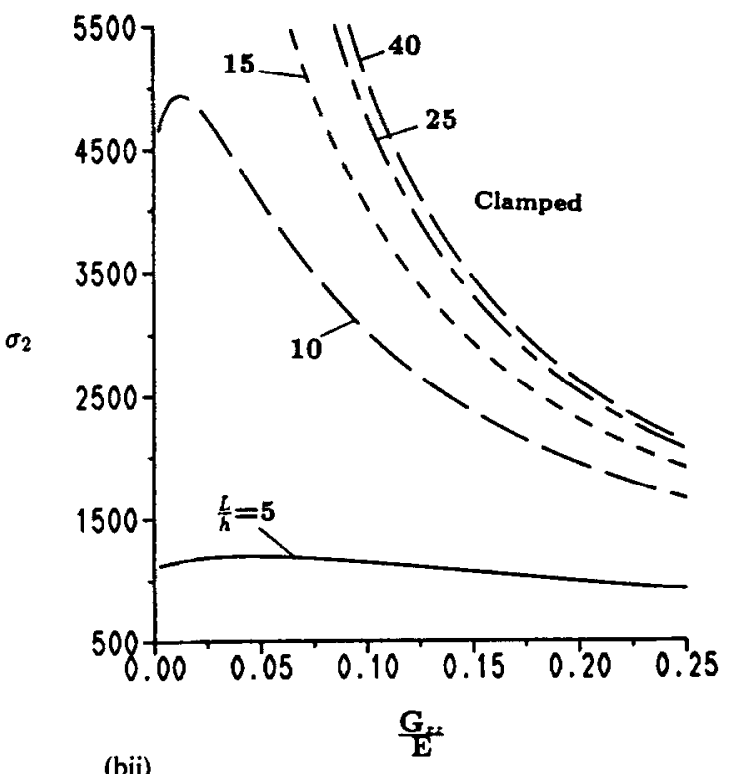

(bii)

Fig. 5. (ai) The effect of $\frac{G_{x z}}{E}$ on $\sigma_{2}$, for pinned-pinned laminated beams. $\frac{E_{z}}{E}=0.1$. (aii) The effect of $\frac{G_{x z}}{E}$ on $\sigma_{2}$, for pinned-pinned laminated beams. $\frac{E z}{E}=1.0$. (bi) The effect of $\frac{G_{x z}}{E}$ on $\sigma_{2}$, for clamped laminated beams. $\frac{E_{z}}{E}=0.1$. (bii) The effect of $\frac{G_{x z}}{E}$ on $\sigma_{2}$, for clamped laminated beams. $\frac{E_{z}}{E}=1.0$.

proceeding to discuss imperfect beam behavior, the value of $\sigma_{2}$ is cross-plotted as a function of beam aspect ratio. These results are shown in Fig. 7(ai), (aii), (bi) and (bii). From these figures, it is seen that for pinned-pinned beams, the initial postbuckling behavior shows a positive slope for beams of aspect ratio in excess of 15 , without regard to the mechanical properties ratios. Clamped beams, however, have a positive $\sigma_{2}$ for all but small values of aspect ratio; this latter situation being limited in addition to very small values of $\frac{E_{z}}{E}$, as infered from Fig. 6(ai) and (aii). 

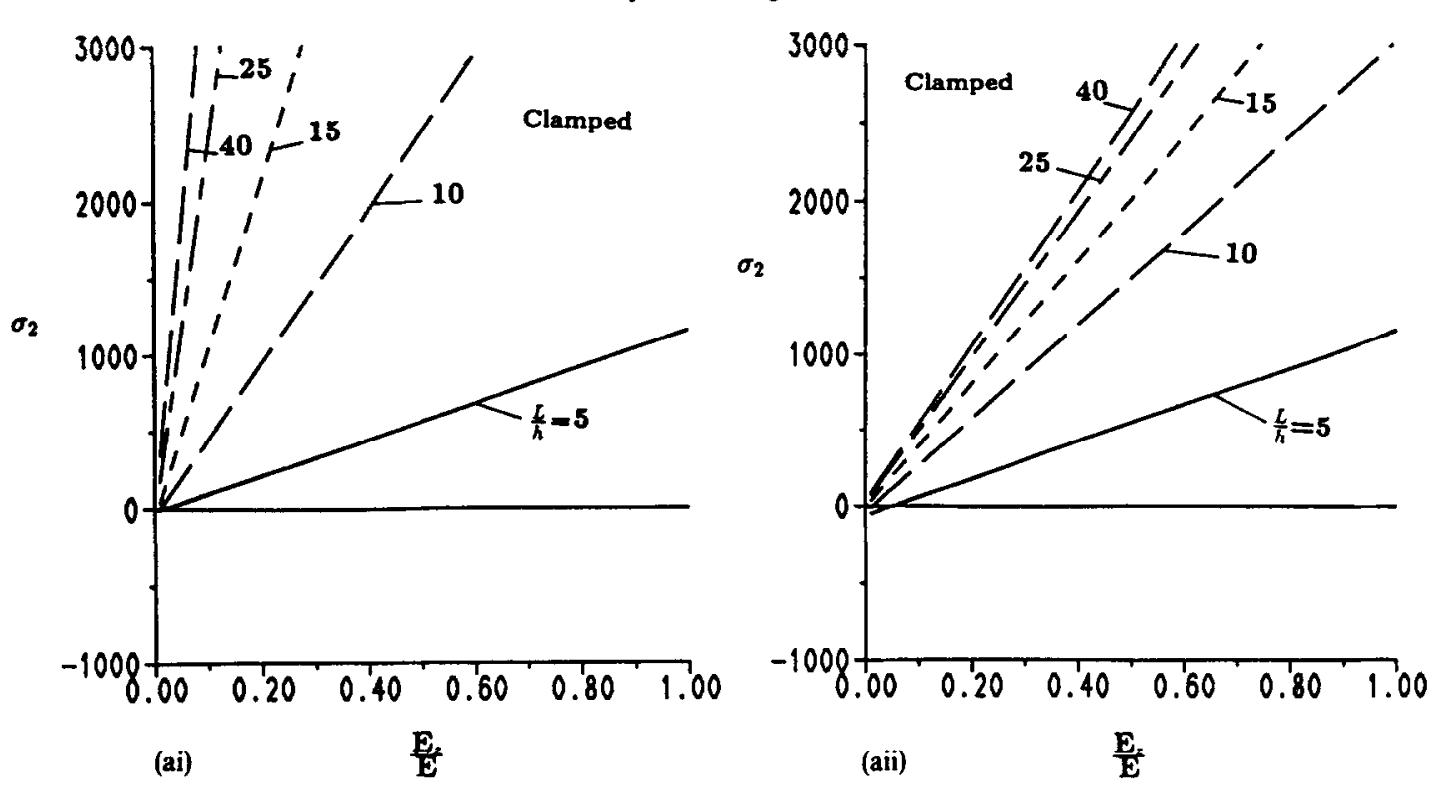

Fig. 6. (ai) The effect of $\frac{E_{z}}{E}$ on $\sigma_{2}$, for clamped laminated beams. $\frac{G_{x z}}{E}=0.01$. (aii) The effect of $\frac{E_{z}}{E}$ on $\sigma_{2}$, for clamped laminated beams. $\frac{G_{x z}}{E}=0.1$.

\section{Initially imperfect beams}

When the beam contains an initial imperfection in shape $\hat{w}(x)$, then the expression for the potential energy needs to be modified, and a procedure similar to that of the perfect case needs to be affected to obtain the response of the beam structure. However, if it is assumed that the imperfection is "small" in the sense that, terms of higher powers of $\hat{w}(x)$ or its derivatives entering in the expression for the potential energy could be neglected, but terms with $\hat{w}(x)$ and $\psi(x)$ (and/or their derivatives) be retained, then the results of the ensuing analysis could be infered from the parameters associated with the preceeding analysis for the perfect cases. This inference was first uncovered by Koiter [13], whose work exemplified the usefulness of an asymptotic postbuckling analysis. Koiter [13] showed that if there was an imperfection in the geometry of the structure of small amplitude $\bar{\varepsilon}$ and in the shape of the buckling mode, and if the corresponding perfect structure obeys the load-deflection result

$$
\sigma=\sigma_{0}+\sigma_{1} \varepsilon+\sigma_{2} \varepsilon+\ldots
$$

then, the load-deflection relationship for the imperfect structure, in the neighborhood of the buckling load of the corresponding perfect structure, is given by ref. [18]

$$
\left(\sigma_{0}-\sigma\right) \varepsilon+\sigma_{1} \varepsilon^{2}+\ldots=\sigma \bar{\varepsilon}
$$

For the isotropic and laminated beam example studied herein, we find that $\sigma_{1}=0$, so that the corresponding imperfect beam obeys the load-displacement relation

$$
\left(\sigma_{0}-\sigma\right) \varepsilon+\sigma_{2} \varepsilon_{+}^{3} \ldots=\sigma \bar{\varepsilon}
$$

Further, for the isotropic case we found $\sigma_{2}>0$, in which case, the response is imperfection insensitive [18]. In the laminated beam example, however, as we have seen, there are some situations corresponding to a particular choice of mechanical properties ratios and geometry, that corresponds to $\sigma_{2}<0$. In such cases, the structure is found to be imperfection sensitive and will reach a maximum load. As an example, consider a pinned TLB, with aspect ratio five and containing a shape imperfection in the form of the fundamental buckling mode and of small amplitude. The load-deflection relation for such a beam, infered from equation (30), valid in the neighborhood of the buckling load of the corresponding perfect beam, is shown in Fig. 8(ai) and (aii), for varying values of non-dimensional imperfection amplitude (normalized by the beam length). This example demonstrates limitload behavior for a beam idealization of a laminate with the inclusion of first-order shear 


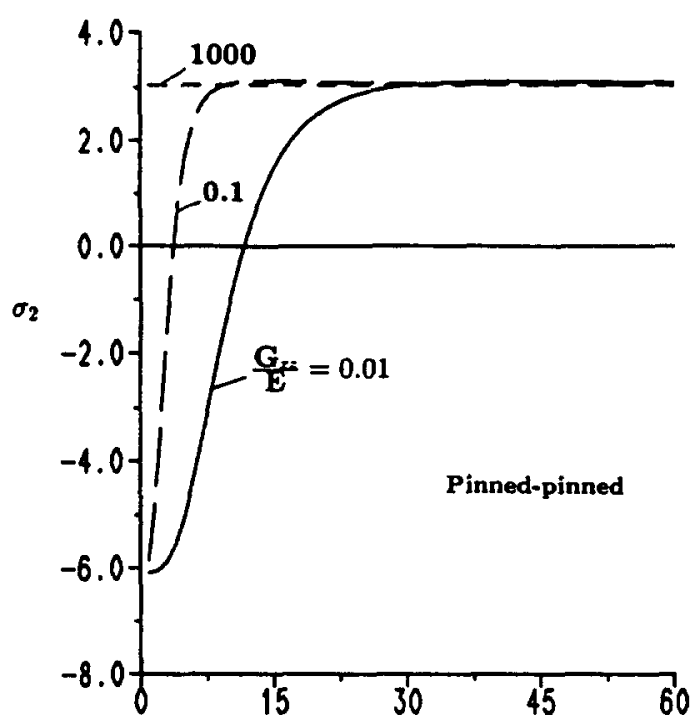

(ai)

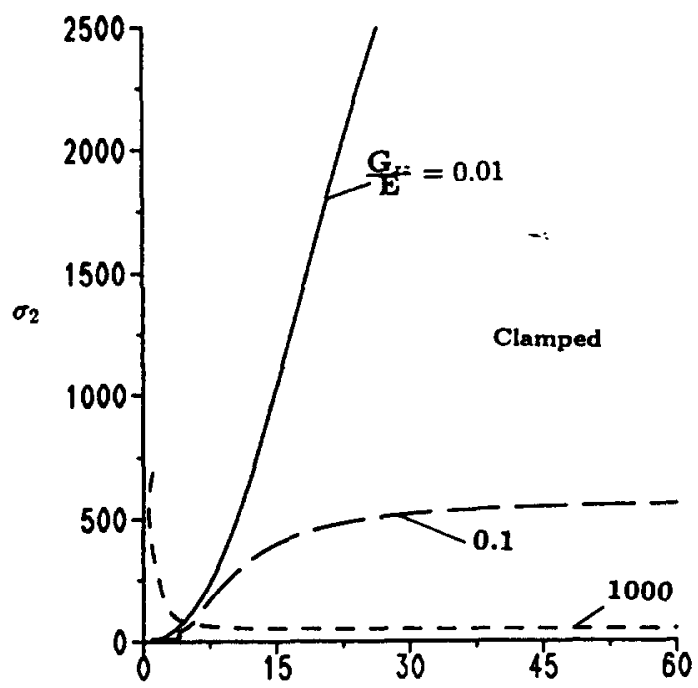

(bi)

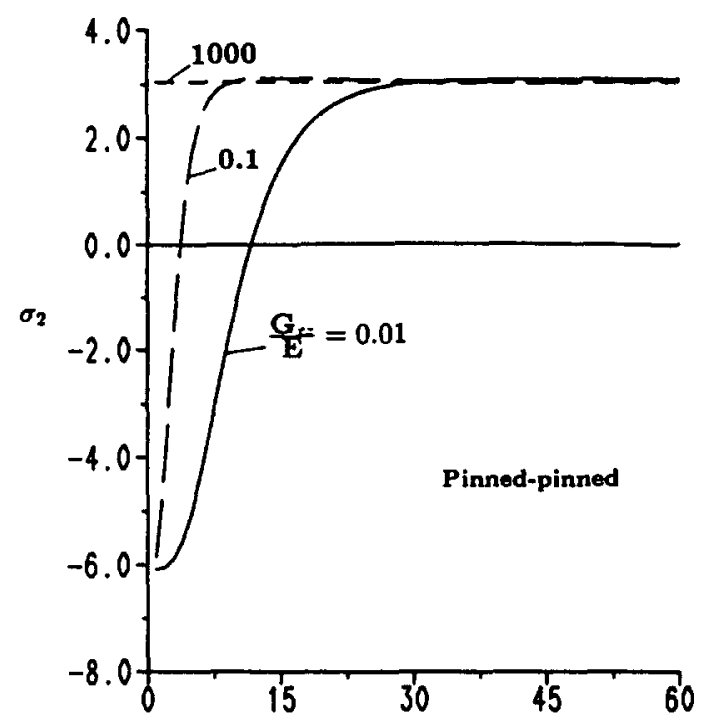

(aii)

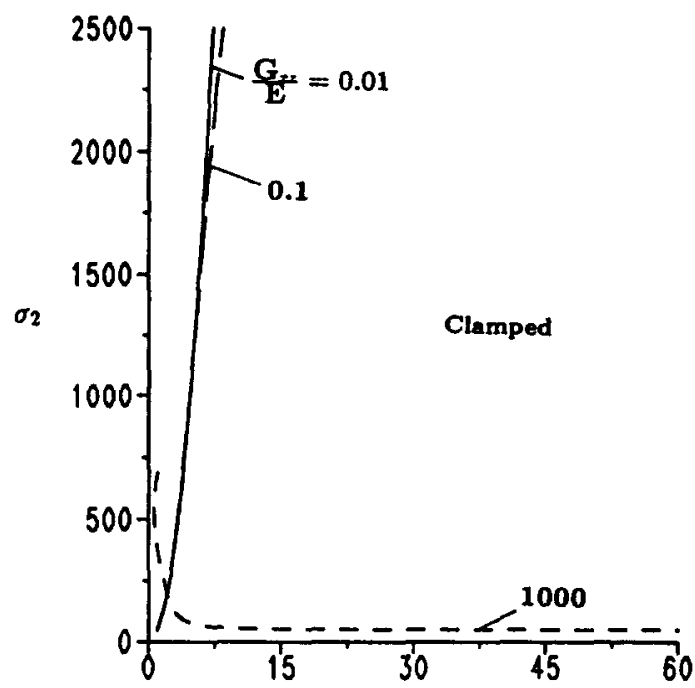

(bii)

\section{$\frac{L}{h}$}

\section{$\frac{L}{h}$}

Fig. 7. (ai) The effect of aspect ratio, $\frac{L}{h}$, on $\sigma_{2}$, for pinned-pinned laminated beams. $\frac{E_{z}}{E}=0.1$. (aii) The effect of aspect ratio, $\frac{L}{h}$, on $\sigma_{2}$, for pinned-pinned laminated beams. $\frac{E_{2}}{E}=1.0$. (bi) The effect of aspect ratio, $\frac{L}{h}$, on $\sigma_{2}$, for clamped laminated beams. $\frac{E_{z}}{E}=0.1$. (bii) The effect of aspect ratio, $\frac{L}{h}$, on $\sigma_{2}$, for clamped laminated beams. $\frac{E_{2}}{E}=1.0$.

deformation effects. From these figures (or using equation (29)), the maximum load carrying capacity of the structure as a function of imperfection size can be obtained. From equation (29), this value is given by

$$
\frac{\sigma_{\max }}{\sigma_{0}}=1-3\left(-\frac{\sigma_{2}}{4}\right)^{1 / 3}(\bar{\varepsilon})^{2 / 3}
$$

Values of $\sigma_{\max }$ corresponding to Fig. 8(ai) and (aii) are tabulated in Table 1. Also given in 


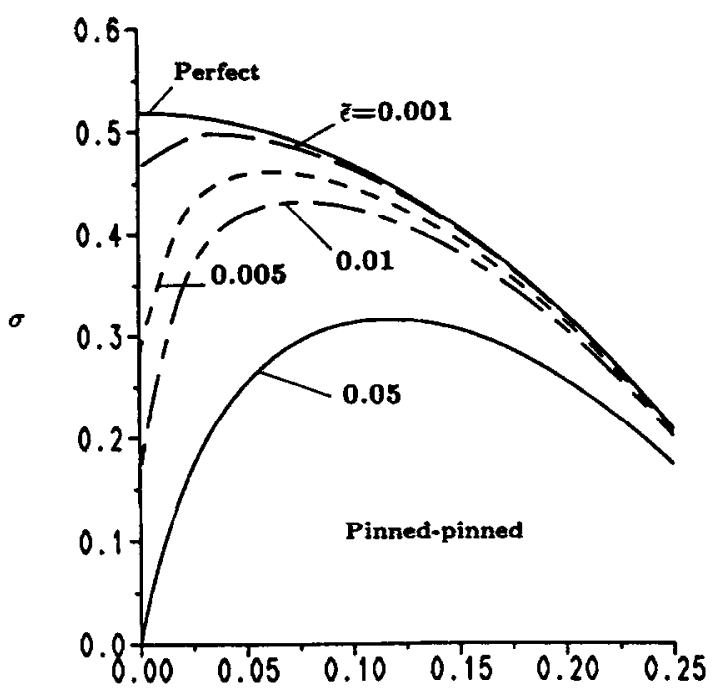

(ai)

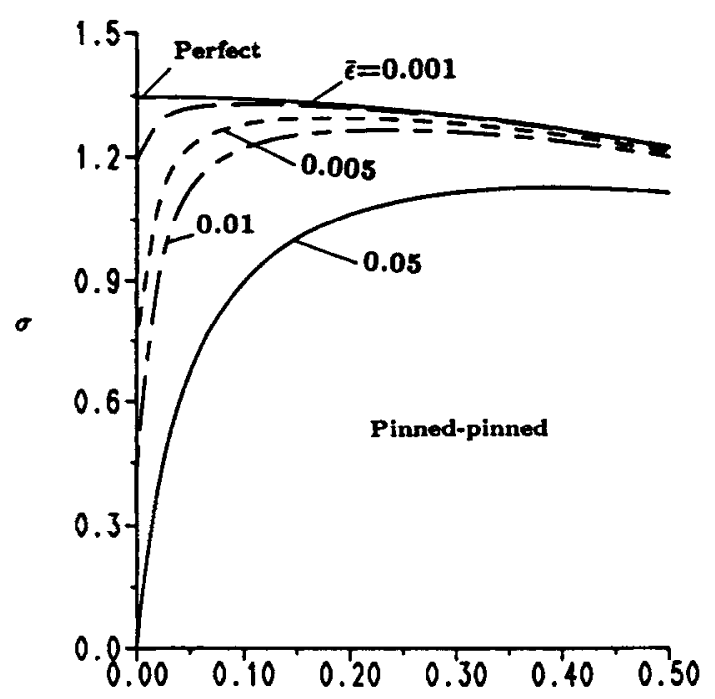

(aii)

Fig. 8. (ai) Effect of imperfections on the postbuckling paths for imperfect pinned-pinned laminated beams (TLB example). $\frac{G_{x z}}{E}=0.011, k=\frac{5}{6}$ and $\frac{L}{h}=5$. (aii) Effect of imperfections on the postbuckling paths for imperfect pinned-pinned laminated beams (TLB example). $\frac{G_{x z}}{E}=0.047$,

$$
k=\frac{s}{6} \text { and } \frac{L}{h}=5 \text {. }
$$

Table 1. Effect of imperfections on maximum load for pinned-pinned TLB with $\frac{L}{h}=5$

\begin{tabular}{|c|c|c|c|c|c|}
\hline $\begin{array}{l}\text { Imperfection } \\
\text { amplitude }\end{array}$ & $\begin{array}{l}\text { Buckling load } \\
\sigma_{0} \\
\text { Figs 8(ai), 8(aii) }\end{array}$ & $\begin{array}{l}\sigma_{\max } \text { corr. } \\
\text { Fig. 8(ai) }\end{array}$ & $\begin{array}{l}\sigma_{\max } \text { corr. } \\
\text { Fig. 8(aii) }\end{array}$ & $\frac{\sigma_{0}-\sigma_{\max }}{\sigma_{0}}$ & $\frac{\sigma_{0}-\sigma_{\max }}{\text { Fig. }^{\sigma_{0}} \text { (aii) }}$ \\
\hline $\begin{array}{l}0.001 \\
0.005 \\
0.01 \\
0.05\end{array}$ & $\begin{array}{l}0.518,1.346 \\
0.518,1.346 \\
0.518,1.346 \\
0.518,1.346\end{array}$ & $\begin{array}{l}0.502 \\
0.470 \\
0.441 \\
0.292\end{array}$ & $\begin{array}{l}1.326 \\
1.288 \\
1.253 \\
1.074\end{array}$ & $\begin{array}{r}3.1 \% \\
9.2 \% \\
14.9 \% \\
43.6 \%\end{array}$ & $\begin{array}{r}1.5 \% \\
4.3 \% \\
6.9 \% \\
20.2 \%\end{array}$ \\
\hline
\end{tabular}

this table are the percent decrease in load carrying capacity for different imperfection amplitudes.

In passing, it is to be noted that a numerical study concerned with postbuckling behavior of laminated beams was reported elsewhere [8]. In that study, the range of examples studied, which were different from those considered here, did not exhibit limit load type behavior.

\section{CONCLUSIONS}

In this paper, we have presented an asymptotic initial postbuckling analysis of pinned-pinned and clamped laminated beams, incorporating first-order shear deformation effects. Expressions derived here for the initial postbuckling response of laminated beams have been shown to reduce to established results, when specialized to isotropic material properties. The coupled influence of mechanical properties and beam aspect ratio is seen to introduce limit load-type behavior for certain ranges of values of these parameters for both pinned-pinned and fully clamped boundary supports. In these cases, the imperfection sensitivity of the beam has been studied and the maximum load carrying capacity calculated 
as a function of normalized imperfection amplitude. Results of a parametric study of the coefficient $\sigma_{2}$, that characterizes the slope of the initial postbuckling load-displacement path has been presented. Mechanical properties of a laminated beam used in tests at the NASA Langley Research Center, and referred to as TLB in this paper, have been used to demonstrate various findings. The applicability of the presented findings seem to be broad. For example, verification of numerical codes written for fully non-linear postbuckling analysis of laminated structures, design and testing of laminated structures etc. The effect of higher-order shear deformation, modeled along the lines of Reddy [21], on the initial postbuckling behavior of laminated beams is currently being pursued, and the results of that investigation will be reported elsewhere.

Acknowledgements -Financial support through a Rackham faculty grant and fellowship from the Graduate School, University of Michigan, Ann Arbor, MI, is gratefully acknowledged.

\section{REFERENCES}

1. J. Whitney and N. J. Pagano, Shear deformation in heterogeneous anisotropic plates. J. appl. Mech. 37, $1031-1036$ (1970).

2. J. N. Reddy, On the generalization of displacement-based laminate theories. Appl. Mech. Rev. 42, 5213-5222 (1989).

3. J. N. Reddy, A review of refined theories of laminated composite plates. Shock Vib. Digest 22, 677-686 (1990).

4. M. Stein, Postbuckling of long orthotropic plates in combined shear and compression. AIAA J. 23, 788-794 (1985).

5. M. Stein, Nonlinear theory for plates and shells including the effects of transverse shearing. AIAA J. 24, $788-794$ (1986).

6. $M$. Stein and D. Jegley, Effects of transverse shearing on cylindrical bending, vibration and buckling of laminated plates. AlAA J. 25, 123-129 (1987).

7. M. Stein, Effects of transverse shearing flexibility on postbuckling of plates in shear. AIAA J. 27, 652-655 (1989).

8. I. Sheinman and M. Adan, The effect of shear deformation on post-buckling behavior of laminated beams. J. appl. Mech. 54, 558-562 (1987).

9. J. N. Reddy and N. D. Phan, Stability and vibration of isotropic, orthotropic and laminated plates according to a higher-order shear deformation theory. J. Sound Vib. 98, 157-170 (1985).

10. N. D. Phan and J. N. Reddy, Analysis of laminated composite plates using a higher order shear deformation theory. Int. J. numer. Meth. Engng 12, 2201-2219 (1985).

11. J. N. Reddy, Analysis of layered composite plates accounting for large deflections and transverse shear strains, in Recent Advances in Non-linear Computational Mechanics (Edited by E. Hinton, D. Owen and C. Taylor), Vol. 1, pp. 155-202 (1982).

12. N. S. Putcha and J. N. Reddy, Stability and natural vibration analysis of laminated plates by using a mixed element based on a refined plate theory. J. Sound Vib. 104, 285-300 (1986).

13. W. Koiter, On the stability of elastic equilibrium. Doctoral thesis, Delft, Amsterdam (1945) (English transiation, AFFDL-TR-70-25, 1970).

14. J. J. Stoker, Nonlinear Elasticity. Gordon and Breach, New York (1968).

15. V. V. Novoshilov, Nonlinear Elasticity. Graylock Press, New York (1953).

16. B. Budiansky and J. W. Hutchinson, Dynamic buckling of imperfection sensitive structures. Proc. 11th Int. Congress of App. Mech, Munich, 1964, pp. 636-651. Springer, Berlin (1966).

17. A. Waas, Initial postbuckling behavior of beams on non-linear elastic foundations. Mech. Res. Commun. 17, 239-248 (1990).

18. C. L. Dym, Stability Theory and its Applications to Structural Mechanics. Noordhoff, Leiden (1974).

19. E. Reissner, The effect of transverse shear deformation on the bending of elastic plates. J. appl. Mech. 12, 69-77 (1945).

20. S. P. Timoshenko and J. M. Gere, Theory of Elastic Stability, 2nd Edition. McGraw Hill, New York (1961).

21. J. N. Reddy, A general non-linear third-order theory of plates with moderate thickness. Int. J. Non-Linear Mech. 25, 677-686 (1990). 